\title{
Fluid flow induced by nonuniform ac electric fields in electrolytes on microelectrodes. III. Observation of streamlines and numerical simulation
}

\author{
N. G. Green, ${ }^{1, *}$ A. Ramos, ${ }^{2}$ A. González, ${ }^{2,3}$ H. Morgan, ${ }^{1}$ and A. Castellanos ${ }^{2}$ \\ ${ }^{1}$ Bioelectronics Research Centre, Department of Electronics and Electrical Engineering, University of Glasgow, Rankine Building, \\ Oakfield Avenue, Glasgow G12 8LT, Scotland, United Kingdom \\ ${ }^{2}$ Departamento de Electrónica y Electromagnetismo, Facultad de Física, Universidad de Sevilla, Reina Mercedes s/n, \\ 41012 Sevilla, Spain \\ ${ }^{3}$ Departamento de Física Aplicada III, ESI Universidad de Sevilla, Camino de los Descubrimientos s/n, 41092 Sevilla, Spain
}

(Received 28 January 2002; published 19 August 2002)

\begin{abstract}
The application of a nonuniform ac electric field to an electrolyte using coplanar microelectrodes results in steady fluid flow. The flow has its origin in the interaction of the tangential component of the nonuniform field with the induced charge in the electrical double layer on the electrode surfaces. Termed ac electro-osmosis, the flow has been studied experimentally and theoretically using linear analysis. This paper presents experimental observations of the fluid flow profile obtained by superimposing images of particle movement in a plane normal to the electrode surface. These experimental streamlines demonstrate that the fluid flow is driven at the surface of the electrodes. Experimental measurements of the impedance of the electrical double layer on the electrodes are also presented. The potential drop across the double layer at the surface of the electrodes is calculated numerically using a linear double layer model, and also using the impedance of the double layer obtained from experimental data. The ac electro-osmotic flow at the surface of the electrodes is then calculated using the Helmholtz-Smoluchowski formula. The bulk fluid flow driven by this surface velocity is numerically calculated as a function of frequency and good agreement is found between the numerical and experimental streamlines.
\end{abstract}

DOI: 10.1103/PhysRevE.66.026305

PACS number(s): 47.65.+a, 82.45.-h, 82.70.Dd, 85.90. + h

\section{INTRODUCTION}

It has been demonstrated that nonuniform ac electric fields generated by coplanar microelectrodes produce steady fluid flow in electrolytic solutions [1-4]. The fluid moves from the high strength field regions on the edges of the electrodes onto the surface of the electrodes, with the highest velocities found at the edge [3]. The flow has been characterized in terms of a number of experimental variables and is dependent on the frequency and amplitude of the applied signal and on the electrolyte conductivity. The velocity goes to zero at high and low frequency limits and is maximum at a frequency that depends on the conductivity and position on the electrode surface [3]. This characteristic frequency is much smaller than the charge relaxation frequency of the bulk electrolyte $f_{c}=\sigma /(2 \pi \varepsilon)$, where $\sigma$ and $\varepsilon$ are the electrical conductivity and permittivity of the electrolyte. Although generated by ac fields, the fluid flow, referred to as ac electro-osmosis, is steady. It can be distinguished experimentally from flow of electrothermal origin because the latter is of smaller magnitude and is mainly observed at frequencies around $f_{c}$ and at higher applied voltages [5-7]. The mechanism of ac electro-osmotic driven fluid flow has recently been shown to be capable of producing unidirectional pumping of liquid on a microscale, as demonstrated experimentally by Brown, Smith, and Rennie [8], following general symmetry arguments presented by Ajdari [9].

The mechanism responsible for the flow is the interaction

*Corresponding author. Email address: n.green@elec.gla.ac.uk of the tangential component of the electric field and the induced charge in the diffuse double layer on the electrode surface $[1,4]$. Consider two coplanar electrodes separated by a thin gap, subjected to an ac potential difference and covered in an electrolyte as shown in Fig. 1. At a certain time, the situation is as shown in the figure; induced charge accumulates in the diffuse double layer with a sign opposite to the electrode charge. This induced charge is subjected to the action of the tangential component of the electric field, giving rise to a force directed from the center of the gap onto the electrode surface. This force drives the fluid at the level of the electrodes and has a direction that is independent of the sign of the electrode potential, so that the fluid flow has a nonzero time average. It should be noted that the mechanism requires a nonuniform electric field thus ensuring that a tangential field component exists in the diffuse double layer on the electrodes.

Other authors have also observed that spatial variations in the normal current distribution on the electrodes cause lateral fluid motion either in ac or dc fields [10,11]. They have suggested that concentration gradients arising from electrode reactions produce a distribution of free charge adjacent to the electrodes; this charge interacts with any lateral electric field generating the electrohydrodynamic flow. Sides [12] further elaborated this theory in order to make predictive calculations for ac applied voltages. In our work, we consider charge transfer reactions to be negligible and that any free charge appears from ideal ion polarization, i.e., the charging of the double layer in the manner of a capacitor in an ac field. The electrodes used in this work were made of Titanium, a metal which readily forms a thin highly resistant oxide layer. Therefore, any Faradaic currents are likely to be extremely 

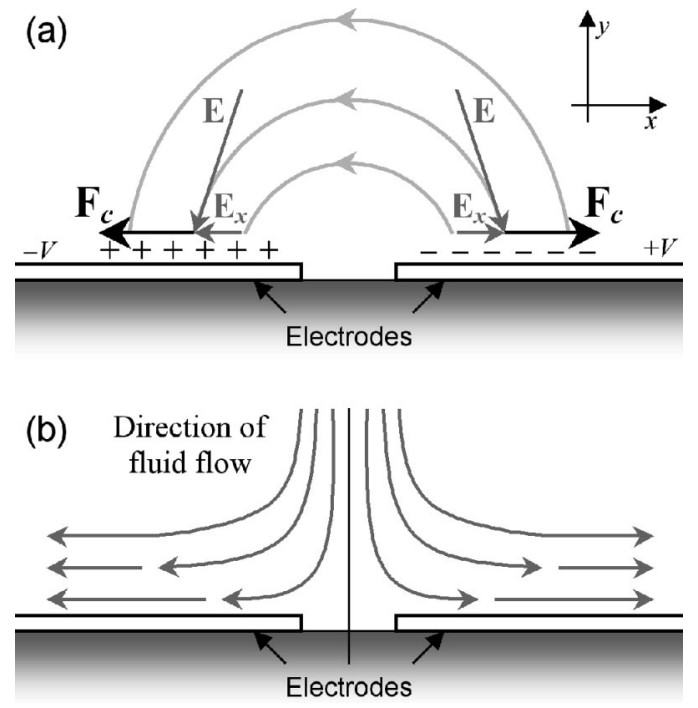

FIG. 1. A schematic diagram of the mechanism of ac electroosmosis for the experimental electrode array, consisting of two long plate electrodes separated by a narrow gap. (a) shows the induced charge layers and the electric field $\mathbf{E}$ at a point in time, resulting from a potential difference applied to the two electrodes. The electric field has a tangential component $\mathbf{E}_{x}$ at the surface of the electrodes, producing a force $\mathbf{F}_{c}$ on the charges at the surface. The time averaged value of this force for an alternating potential is nonzero, producing the steady fluid flow pattern shown schematically in (b). The fluid flow is driven at the surface of the electrode, moving out across the surface and dragging fluid down in the center of the gap.

small and are not considered in the analysis.

The electro-osmotic velocity has been calculated using a simple circuit model to represent the fluid/interface system [1], as well as the linear analysis of the electrokinetic equations [4]. Both models have a good degree of correlation with experimental data, predicting that the frequency dependence of the fluid flow is governed by electrode polarization, as demonstrated experimentally [3].

This paper presents experimental results of the flow streamlines imaged in a plane normal to the surface of the microelectrodes. The streamlines were obtained by a superposition of several images of latex tracer particles moving with the fluid. In order to determine the specific impedance of the double layer at the surface of the electrodes, experimental measurements were made of the frequency-dependent impedance of the electrode/electrolyte system.

Finally, the solution for the ac electro-osmotic driven fluid flow is calculated numerically using the finite element method. First the electric potential was solved using one of two boundary conditions at the electrode surface; either using the double layer capacitance determined from the linear Debye-Hückel model [13] or using the experimentally determined values for the double layer impedance. The electrical stress on the fluid and the fluid velocity at the surface were then calculated and compared with experimental measurements [3]. Finally, the motion of the bulk fluid was numerically calculated, using the ac electro-osmotic velocity on the electrode surface as the boundary condition. These results

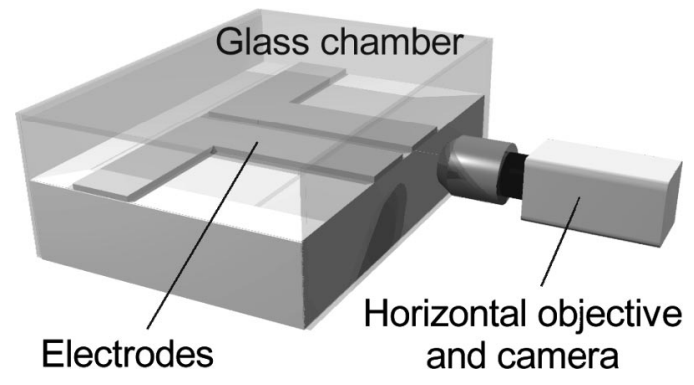

FIG. 2. A schematic diagram of the experimental setup. The electrodes were fabricated up to the edge of the substrate and a glass chamber was constructed around the array, with a vertical glass plate at the end of the electrodes. A microscope objective and camera were then placed horizontally looking along the gap between the electrodes. The camera was then focused at a point inside the chamber at sufficient distance from the electrode ends so that the fluid flow was moving in the vertical plane of focus only.

were compared with the experimental observations of the streamlines.

\section{EXPERIMENTAL RESULTS}

The fluid motion has been measured in detail as a function of the applied field frequency, voltage, and medium conductivity [3]. In general, the fluid velocity increases as the voltage is increased and decreases with increasing electrolyte conductivity. The magnitude of the velocity as a function of frequency has a bell-shaped profile and tends to zero at high and low frequency limits.

\section{A. ac electro-osmotic streamlines}

The streamlines were recorded using an experimental setup that has been described previously [14], a schematic of which is shown in Fig. 2. In summary, two coplanar bar electrodes, $0.5 \mathrm{~mm}$ wide, $2 \mathrm{~mm}$ long, and $120 \mathrm{~nm}$ thick, with their long edges parallel and separated by $25 \mu \mathrm{m}$, were microfabricated on a glass slide. The electrodes consisted of a layer of gold sandwiched between two layers of titanium. The electrodes were coated in a thin titanium layer because of the well known resistance of this metal to corrosion in air or in saline solutions [15]. Titanium forms a very thin oxide layer that usually renders it inactive. Therefore, to a good approximation, the electrodes can be considered to be perfectly polarizable in the range of applied voltages $(0-2.5 \mathrm{~V})$. A square glass chamber was constructed around the electrode array, so that the electrode/electrolyte system could be observed both from above and from the side. A microscope objective and camera were pointed horizontally along the electrodes with the gap between the electrodes in the center of the field of view, so that the electrodes could be imaged in cross section [14]. An ac potential difference was applied to the pair of electrodes with voltage amplitude from 0 to $2.5 \mathrm{~V}$, and frequencies ranging from $10^{2}$ to $10^{5} \mathrm{~Hz}$. This frequency range is always below the charge relaxation frequency $f_{c}$ $=\omega_{c} / 2 \pi=(1 / 2 \pi)(\sigma / \varepsilon)$, which is from $10^{6}$ to $10^{8} \mathrm{~Hz}$ for the experimental conductivities. 
The electrolytic solutions used, both for the study of the fluid motion and the measurement of the double layer impedance, were aqueous solutions of potassium chloride $(\mathrm{KCl})$ with three different conductivities: 2.1 (hereafter referred to as case $A$ ), 8.6 (case $B$ ), and $84 \mathrm{mS} / \mathrm{m}$ (case $C$ ). Fluorescent latex spheres (Molecular Probes, Oregon, U.S.), $557 \mathrm{~nm}$ in diameter, were used as tracer particles $[3,14]$. The movement of the particles was recorded on video and transferred to computer. Successive video frames were superimposed to produce tracks of particle movement over time. In the absence of other forces, the particles are small enough to follow the fluid so that the particle tracks can be considered to be the streamlines of the fluid.

Three composite images of particle tracks are shown in Figs. 3(a), 3(b), and 3(c) for different frequencies (100, 300, and $1000 \mathrm{~Hz}$, respectively), for electrolyte $A$. There is some dielectrophoretic capture $[16,17]$ of particles at the edges of the electrodes and some collection of particles on top of the electrodes (white regions). This latter effect could be caused by dipole-dipole interparticle interaction, or particle-particle, particle-electrode interactions of electrohydrodynamic nature not well understood at present $[10,11,18]$. However, it is clear that in the bulk, the particle tracks show that the fluid circulates in two symmetrical rolls with the fastest velocities close to the electrode edges. Particle tracks indicate that the fluid moves from the center top of the image, down to the center of the gap and out across the electrodes as shown schematically in Fig. 1.

The highest velocities were observed close to the electrode surface, a good indication that the fluid flow is driven in this region. The three images were taken at frequencies where the fluid flow can clearly be observed. The pattern of the fluid flow depends on the frequency of the applied signal. At $1 \mathrm{kHz}$ [Fig. 3(c)] the velocity is high at the electrode edge and decreases rapidly with increasing distance from the edge. This produces a flow that has a center of circulation close to the electrode edge and movement that is restricted to the region close to the gap between the electrodes. Particles farther away (at the edge of the image) move more slowly and demonstrate Brownian motion; the broken (jiggling) tracks are an indication of this. As the frequency is decreased [Figs. 3(b) and 3(a)], the fluid velocity at the electrode edge remains constant or decreases slightly. However, the rate of decrease of velocity with distance over the electrodes is less, resulting in the center of the streamlines moving farther away from the electrode edge over the surface.

\section{B. Double layer impedance measurements}

In order to determine the frequency dependence of the fraction of the total applied voltage drop across the double layer, double layer impedance measurements were made using a specially constructed measurement cell. The measurement cell consisted of two large $\left(35.5 \times 4.5 \mathrm{~mm}^{2}\right)$ parallel plate electrodes fabricated on planar glass slides, separated using a thin spacer of $0.7 \mathrm{~mm}$ thickness. The electrodes were made of the same sandwich of titanium/gold/titanium as those used for fluid flow observations. The impedance of the electrode/electrolyte system was measured using a Hewlett
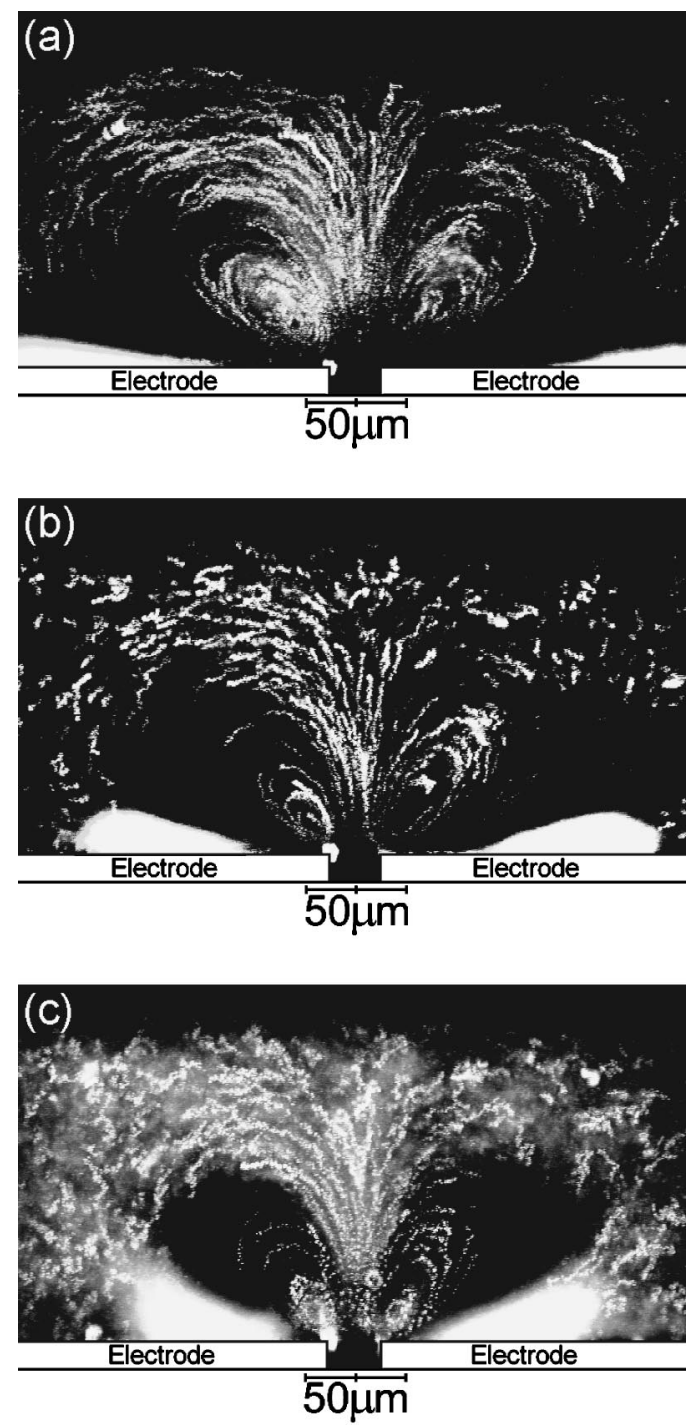

FIG. 3. Composite images of particle tracks over time, obtained by superimposing successive video frames from footage of fluid flow observed using the experimental setup shown in Fig. 2, for three different frequencies of the applied potential: (a) 100, (b) 300, and (c) $1000 \mathrm{~Hz}$. The amplitude of the applied voltage was $2 \mathrm{~V}$ in all cases and the suspending medium was electrolyte $A$. As the frequency increased, the rolls in the fluid flow extended a smaller distance over the surface of the electrodes. The center of the rolls also moved close to the surface and the edge of the electrodes.

Packard impedance analyzer 4192A with the signal applied to one electrode. The second electrode was attached to ground to complete the circuit. The cell was filled with $\mathrm{KCl}$ solutions of varying conductivities and the impedance of the cell was measured as a function of frequency and voltage of the applied signal.

The total impedance of the sample $Z_{T}$ can be represented as a parallel RC circuit with frequency-dependent resistance and capacitance defined by

$$
Z_{T}=\frac{R}{1+i \omega C R} .
$$

Figure 4 shows a plot of $R$ and $C$ as a function of frequency, 


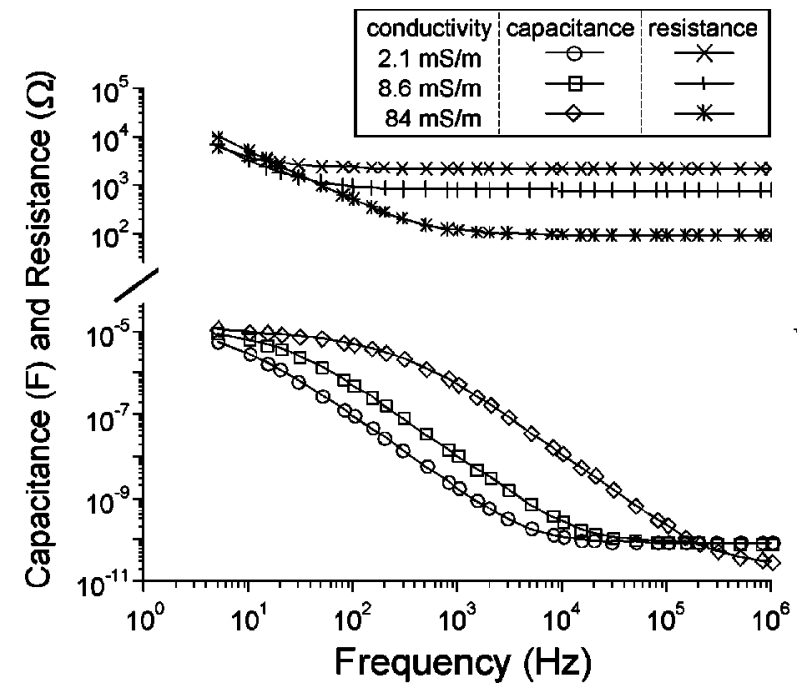

FIG. 4. The capacitance and resistance for the three electrolytes $A, B$, and $C$ measured using two parallel plate electrodes for an applied potential of amplitude $0.5 \mathrm{~V}$ and plotted as a function of frequency.

at an applied voltage of $0.5 \mathrm{~V}$ for three electrolyte conductivities as used in a previous work [3]. This data shows that the capacitance and resistance are functions of frequency. At high frequencies, the capacitance tends to a constant value equivalent to that of a parallel plate capacitor with the permittivity of the bulk electrolyte. The resistance is also constant at high frequencies, with a value that is inversely proportional to the conductivity of the solution. These limiting values correspond to the impedance of the bulk electrolyte, since at high frequencies the double layer impedance is negligible. At low frequencies, the total measured resistance and capacitance includes the impedance of the double layer. Assuming that the impedance of the double layer and the bulk are in series, the bulk impedance can be subtracted from the total impedance to obtain values for the frequency-dependent double layer impedance $Z_{\mathrm{DL}}$. The double layer impedance determined using this method is shown in Fig. 5 for electrolyte $A$ and a voltage amplitude of $0.5 \mathrm{~V}$.

The results show that the double layer polarization imped-

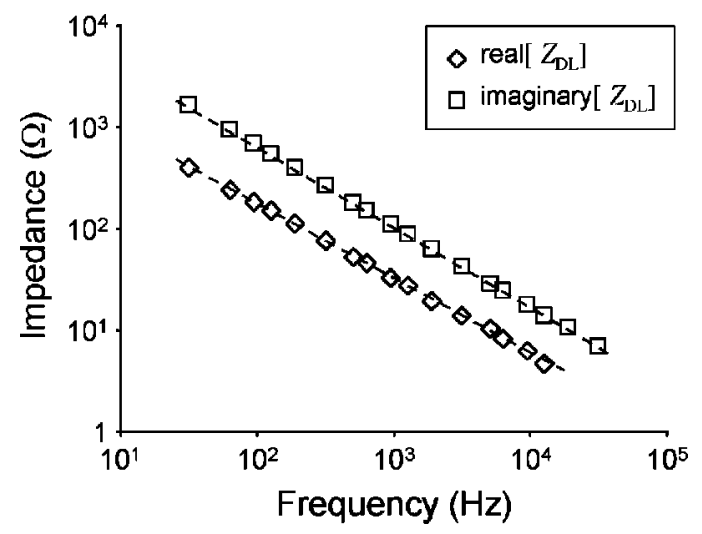

FIG. 5. The real and imaginary components of the double layer impedance for electrolyte $A$ and $0.5 \mathrm{~V}$, obtained by subtracting the bulk impedance values from the measured impedance. ance can be fitted to the following expression:

$$
Z_{\mathrm{Dl}}=\frac{A}{(i \omega)^{\beta}}=\frac{A}{\omega^{\beta}}\left[\cos \left(\frac{\pi}{2} \beta\right)-i \sin \left(\frac{\pi}{2} \beta\right)\right],
$$

where $A$ and $\beta$ are constants. This type of polarization impedance is frequently found in double layer measurements and is referred to as constant phase angle (CPA) impedance [15,19-23]. This expression satisfies the Kramers-Kronig relations for the impedance of a linear system [24]. For a perfect capacitor, $\beta=1$ and for a perfect resistor, $\beta=0$. For double layer impedance measurements on solid electrodes, $\beta$ is typically found to be between 0.7 and 0.9 [20]. An analysis of the measurements performed in this work gave values of $\beta$ around $0.80 \pm 0.05$, depending on conductivity and voltage. The voltage dependence of the impedance indicates a nonlinear system, as observed by other researchers [25]. As an example, for the three different electrolyte conductivities used in this work and an applied voltage of amplitude $0.5 \mathrm{~V}$, the values of $\beta$ were $0.75,0.78$, and 0.8 . The corresponding values for the constant $A$ were 18000,18400 , and 19000 with $\omega$ in $\operatorname{rads}^{-1}$ and $Z$ in ohms. At the lower voltage amplitude of $0.1 \mathrm{~V}$, the values of $\beta$ were $0.80,0.80$, and 0.82 for electrolytes $A, B$, and $C$, respectively. The corresponding values for the constant $A$ were 27000,22000 , and 23500.

For the ac electro-osmotic flow measurements [3], the double layer impedance measurements and fluid flow observations, the frequency of the applied signal was much lower than the charge relaxation frequency, $f_{c}$. In this lower frequency range, the experimental results indicate that the double layer is not purely capacitive (shown by the deviation of $\beta$ from unity) as expected from theory [26]. The nonideal behavior of the double layer cannot be ascribed to ionic diffusion effects since the standard differential capacitance of the double layer is not modified in this frequency range [26]. The explanation for the nonunity value of $\beta$ is a topic of considerable current research $[19,22,23]$, and a number of possible theories have been proposed, including surface disorder such as atomic-scale heterogeneities, or geometric irregularities (roughness).

However, even though there is no well-defined physical explanation, the measured CPA impedance can provide a useful means of modelling the impedance of the system. The specific impedance of the double layer can be obtained by multiplying the double layer impedance by the area of the electrode $\left(35.5 \times 4.5 \mathrm{~mm}^{2}\right)$. This figure gives the ratio between the potential drop and the current density, which, in turn, can be used to define a boundary condition for the numerical simulation that may be more valid for comparison with the experimental results.

\section{THEORETICAL ANALYSIS}

In this section the equations and boundary conditions governing the electrical potential and the fluid velocity will be described. The subsequent section presents the results of the numerical simulation of the governing equations for the experimental electrode system. 


\section{A. The physical system}

As described earlier, the experimental system consists of two long thin coplanar parallel electrodes fabricated on a glass slide, as shown schematically in Fig. 2. Above the surface, there is a solution of $\mathrm{KCl}$ bounded by an upper horizontal glass cover slip. At the glass/electrolyte and metal/ electrolyte interfaces, double layers are formed with a typical thickness given by the Debye length,

$$
\lambda_{D}=\sqrt{\frac{D \varepsilon}{\sigma}},
$$

where $D$ is the mean diffusion coefficient of the ions. The Debye length is negligibly small compared to the typical size of the system $L\left(L \sim 10 \mu \mathrm{m}, \sigma \sim 10^{-2} \mathrm{~S} / \mathrm{m}, \lambda_{D} / L \sim 10^{-3}\right)$. Since the electrodes are long $(\sim 2 \mathrm{~mm})$, both compared to gap width $(\sim 25 \mu \mathrm{m})$ and the size of the roll observed in the fluid $(\sim 100 \mu \mathrm{m})$, the problem can be considered to be two dimensional.

When an ac voltage is applied to the electrodes, an electrical current is established in the solution. The following analysis assumes that electrolysis does not occur at the electrode surfaces, i.e., the electrodes are considered to be perfectly polarizable, and the double layer behaves in a linear manner.

\section{B. The electrical potential}

As previously stated, for sufficiently low frequencies, i.e., $f \ll f_{c}$, the double layer is in quasiequilibrium [26]. Under these conditions, the bulk electrolyte behaves in a resistive manner and the double layer in a capacitive manner. As a result, the potential in the bulk electrolyte satisfies Laplace's equation

$$
\nabla^{2} \phi=0
$$

with the boundary condition just outside the double layer on the electrode surface given by

$$
\sigma \frac{\partial \phi}{\partial y}=\frac{\partial q_{\mathrm{DL}}}{\partial t}
$$

where $q_{\mathrm{DL}}$ is the charge per unit area in the double layer. In this equation we assume that lateral currents along the double layer are negligible. Equation (5) describes the charging of the double layer due to the bulk current. The relationship between the charge and the potential drop across the double layer depends on the model used. If the voltage drop across the diffuse double layer is sufficiently small $\left(\Delta \phi_{d}\right.$ $<k T / e=0.025 \mathrm{~V}$ ) there is a linear relationship between the charge and the voltage, i.e., $q_{\mathrm{DL}}=C_{\mathrm{DL}}(\phi-V)$, and the equation can be written with complex amplitudes as

$$
\sigma \frac{\partial \phi}{\partial y}=i \omega q_{\mathrm{DL}}=i \omega C_{\mathrm{DL}}\left(\phi-V_{j}\right),
$$

where $C_{\mathrm{DL}}$ is the capacitance per unit of area of the total double layer (diffuse plus compact layers), and $V_{j}$ is the potential applied to the electrode $j$. This capacitance $C_{\mathrm{DL}}$ is given by the series combination of the Stern or compact layer capacitance, $C_{s}$, and the diffuse double layer capacitance, $C_{d}$,

$$
C_{\mathrm{DL}}=\frac{C_{s} C_{d}}{C_{s}+C_{d}}
$$

The compact layer capacitance is attributed almost entirely to the oxide layer which forms on top of the titanium electrode and is approximately independent of the electrolyte concentration. The properties of the diffuse part of the double layer depend on electrolyte concentration as given by the Gouy-Chapman theory [13]. Although experimentally the potential drop across the diffuse double layer can exceed $0.025 \mathrm{~V}$, nevertheless the linear analysis can give useful information into the mechanism governing fluid flow.

At the interface between the electrolyte and the glass, a similar boundary condition holds. However, the boundary condition can be simplified in this case. In the absence of lateral currents through the double layer, the total normal current, free plus displacement, must be continuous. Therefore, the total normal current in the electrolyte and the glass are equal,

$$
(i \omega \varepsilon+\sigma) \frac{\partial \phi}{\partial y}=\left(i \omega \varepsilon_{G}+\sigma_{G}\right) \frac{\partial \phi_{G}}{\partial y}
$$

where $\varepsilon_{G}, \sigma_{G}$, and $\phi_{G}$ are the electrical permittivity, conductivity, and potential in the glass. Since the angular frequency $\omega \ll \sigma / \varepsilon<\sigma / \varepsilon_{G}$, and since the conductivity of the glass is negligible, the boundary condition at the glass interface in the fluid, Eq. (8), simplifies to

$$
\frac{\partial \phi}{\partial y}=0
$$

In deriving these boundary conditions, the presence of lateral currents along the double layer has been neglected. In this theoretical analysis, the applied and natural surface potentials are assumed to be small. In this case, as discussed by González et al. [4], the intrinsic mean ion density of the diffuse double layer is small and the lateral currents are negligible. For high ion concentrations in the double layer, surface currents might be comparable to the currents in the normal direction.

Owing to electrode polarization, the electric field in the bulk electrolyte is frequency dependent. When the frequency is low, most of the applied voltage is dropped across the double layer but when the frequency is high most of the applied voltage is dropped across the bulk electrolyte. The typical angular frequency of transition can be estimated from simple circuit theory as $\omega_{0} \sim(R C)^{-1}=\sigma /\left(C_{\mathrm{DL}} L\right)$, where $L$ is a typical size of the system, for example, the electrode gap $[1,2]$. The capacitance per unit area can be estimated from the Debye-Hückel theory as $\varepsilon / \lambda_{D}$. The approximate transition frequency is therefore, $\omega_{0} \sim(\sigma / \varepsilon)\left(\lambda_{D} / L\right)$, which is several orders of magnitude smaller than the charge relax- 
ation frequency, $\sigma / \varepsilon$, of the electrolyte. The fluid flow is observed at frequencies of the order of the transition frequency $\omega_{0}[1,3,8]$.

\section{The fluid dynamic problem}

Once the potential is solved, the electro-osmotic velocity just outside the double layer at the surface of the electrodes can be calculated from this solution.

\section{The solution in the diffuse layer}

The fluid motion is caused by electrical stresses that are nonzero only in the diffuse double layer, since the charge density in the bulk is zero. These stresses result in a rapidly varying velocity profile in the diffuse double layer, changing from zero at the wall to a finite value just outside the double layer. This velocity value can be used as a boundary condition at the electrode surface to calculate the bulk motion. In the thin double layer approximation, for diffuse layers in quasiequilibrium (low frequency compared to $\sigma / \varepsilon$ ) and on a perfectly polarizable metal surface, the slip electro-osmotic velocity is given by the Helmholtz-Smoluchowski formula [27],

$$
u=-\frac{\varepsilon}{\eta} \Delta \phi_{d} \frac{\partial \phi}{\partial x}=\frac{\varepsilon}{\eta} \Delta \phi_{d} E_{x} .
$$

In this expression, $E_{x}$ is the tangential field just outside the diffuse layer, $\eta$ is the fluid viscosity, and $\Delta \phi_{d}=\phi-\psi$ represents the difference between the potential $\phi$ on the outer side of the diffuse layer and the potential $\psi$ on the inner side of this layer, at the nonslip plane. This equation gives the tangential velocity on the electrodes; the normal velocity is zero.

In the linear regime, the time-averaged horizontal fluid velocity at the interface between the double layer and the bulk is [4]

$$
\begin{aligned}
\langle u\rangle & =-\frac{\varepsilon}{2 \eta} \operatorname{Re}\left[\Delta \phi_{d} \frac{\partial \phi^{*}}{\partial x}\right] \\
& =-\frac{\varepsilon}{2 \eta} \Lambda \operatorname{Re}\left[\Delta \phi_{\mathrm{DL}} \frac{\partial \phi^{*}}{\partial x}\right] \\
& =-\frac{\varepsilon}{4 \eta} \Lambda \frac{\partial}{\partial x}\left|\phi-V_{j}\right|^{2},
\end{aligned}
$$

where $\operatorname{Re}[B]$ means real part of $B$, the asterisk indicates complex conjugate, $\Lambda$ is the ratio between the diffuse layer potential drop $\Delta \phi_{d}$ and the total double layer potential drop $\Delta \phi_{\mathrm{DL}}$, and $V_{j}$ is the potential applied to the electrode. The parameter $\Lambda$ is given by the ratio between the total capacitance $C_{\mathrm{DL}}$ and the diffuse double layer capacitance,

$$
\Lambda=\frac{C_{\mathrm{DL}}}{C_{d}}=\frac{C_{s}}{C_{s}+C_{d}} .
$$

Since $\Lambda<1$, the presence of the extra capacitance due to the compact layer decreases the maximum achievable slip velocity. Equation (11) gives the boundary condition for the tan- gential velocity on the electrodes. For the glass/electrolyte interface an estimate of the potential drop across the diffuse double layer shows that the tangential velocity here is negligibly small. At the boundary

$$
i \omega C_{\mathrm{DL}}\left(\phi-\phi_{G}\right)=i \omega \varepsilon_{G} \frac{\partial \phi_{G}}{\partial y},
$$

so that the potential drop across the diffuse layer on the glass is

$$
\phi-\phi_{G}=\frac{\varepsilon_{G}}{C_{\mathrm{DL}}} \frac{\partial \phi_{G}}{\partial y} \sim \frac{\varepsilon_{G} \lambda_{D}}{\varepsilon L} \phi_{G},
$$

which is of the order $\lambda_{D}$ divided by the typical distance $L$ of the system. Therefore, from Eq. (10), the electro-osmotic velocity on the glass is negligible.

\section{The solution in the bulk}

To obtain the velocity in the bulk, the Navier-Stokes equations must be solved. Since, for microelectrodes the Reynolds number is usually very small, $\rho_{m} u L / \eta \leqslant 10^{-2}$, we neglect the inertial terms in the Navier-Stokes equations. In the absence of externally applied body forces, these equations reduce to

$$
\eta \nabla^{2}\langle\mathbf{u}\rangle-\nabla p=0, \quad \boldsymbol{\nabla} \cdot\langle\mathbf{u}\rangle=0 .
$$

The boundary conditions are (1) the tangential ac electroosmotic velocity on the electrodes, Eq. (11); (2) zero tangential velocity on the glass; and (3) zero normal velocity on every boundary.

In addition to the time-averaged velocity there will also be an alternating velocity, resulting from the oscillating part of the electrical force in the diffuse double layer. This oscillating electro-osmotic velocity diffuses into the bulk up to distances of the order $\left[\eta /\left(\rho_{m} \omega\right)\right]^{1 / 2}[9]$. This velocity is only observed in our experiments for very low frequencies $(1-10$ $\mathrm{Hz}$ ), since the amplitude of the oscillation of the tracking particles is too small at higher frequencies.

\section{Calculation of ac electro-osmotic flow using the experimental polarization impedance}

The measured polarization impedance of the metal/ electrolyte interface provides an empirical relationship between the potential drop across the double layer and the electrical current. The frequency dependence of the ac electroosmotic fluid flow is closely correlated with the mechanism of electrode polarization $[1,3]$. It can be concluded therefore that a phenomenological model for ac electro-osmosis based on the measured impedance of the double layer will give a better description of the fluid flow.

In this case the electrical potential in the bulk is a solution of Laplace's equation with a new boundary condition at the electrode surface given by

$$
\sigma \frac{\partial \phi}{\partial y}=\frac{1}{Z_{\mathrm{DL}}}\left(\phi-V_{j}\right),
$$


where $Z_{\mathrm{DL}}$ is the measured specific impedance of the double layer (Sec. II B). We assume that Eq. (11) is valid even if the measured impedance of the double layer is not purely capacitive. The time-averaged slip velocity is then given by

$$
\langle u\rangle=-\frac{\varepsilon}{4 \eta} \Lambda \frac{\partial}{\partial x}\left|\phi-V_{j}\right|^{2},
$$

with $\phi$ given by the solution of the electric potential with the new boundary condition, Eq. (16). This expression for the velocity was derived from the Smoluchowski expression [Eq. (10)] for the ideal model of a compact Stern layer in series with a diffuse layer. In this ideal model, $\Lambda$ is given by Eq. (12) and indicates how much of the potential drop across the double layer, $\Delta \phi_{\mathrm{DL}}$, is dropped across the diffuse layer, $\Delta \phi_{d}$, i.e., $\Lambda=\Delta \phi_{d} / \Delta \phi_{\mathrm{DL}}$. In our phenomenological model for a real double layer, $\Lambda$ is assumed to be a measure of this ratio.

In the numerical section of this paper (Sec. IV), the velocities calculated using Eq. (17) with the experimental impedance boundary condition will be compared with those calculated using the double layer capacitance given by the Debye-Hückel theory.

\section{NUMERICAL RESULTS AND DISCUSSION}

Numerical calculations were performed using FLEXPDE (PDE Solutions) [28], a commercially available finite element partial differential equation solver. In general, a twodimensional (2D) problem space is divided into triangular elements and the variables are approximated by second or third order polynomials in each element. In order to solve the Navier-Stokes equation, the penalty method described in Ref. [29] was used.

The problem space for the numerical simulation is shown schematically in Fig. 6. Owing to the symmetry of the electrodes, the problem space can be restricted to one electrode of the two with appropriate symmetry boundary conditions. The inner edge of the electrode is $12.5 \mu \mathrm{m}$ from the symmetry boundary, giving an interelectrode separation of $25 \mu \mathrm{m}$. The height of the solution space is $200 \mu \mathrm{m}$, corresponding to the height of the fluid filled chamber; the length of the solution space was much larger than the gap and the electrode.

\section{A. Numerical simulation of the electrical potential and calculation of ac electro-osmotic velocity on the surface of the electrodes}

The electric potential satisfies Laplace's equation [Eq. (4)] with the following boundary conditions. On the surface of the electrodes, we have from Eq. (16), which is an extension of Eq. (6),

$$
\sigma Z_{\mathrm{DL}} \frac{\partial \phi}{\partial y}=\phi-V
$$

In the numerical solution, two versions of this boundary condition are considered. First, using the linear Debye-Hückel model, the impedance corresponds to a capacitor; $Z_{\mathrm{DL}}$ $=1 / i \omega C$, with $C=\varepsilon / \lambda_{D}$. In this case, the problem needs

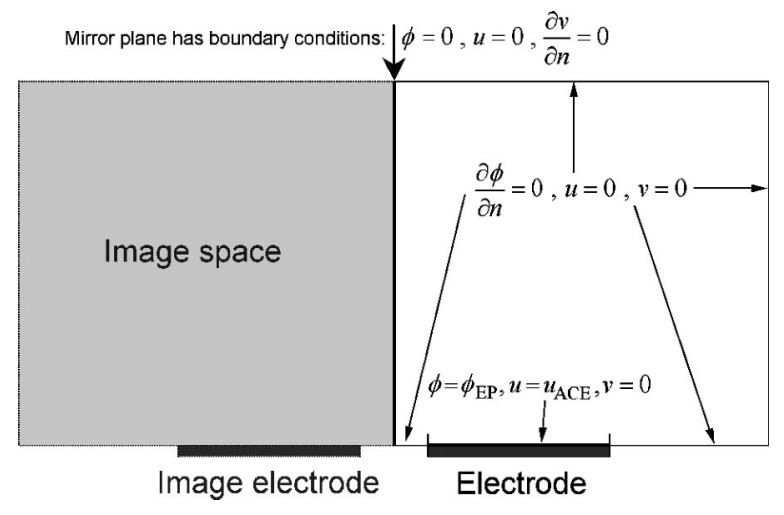

FIG. 6. A schematic diagram of the $2 \mathrm{D}$ problem space for the numerical solution of the electrical potential $\phi$ and the fluid flow $\mathbf{u}=(u, v)$. The boundary condition for the potential on the electrode was $\phi_{\mathrm{EP}}=V+\sigma Z_{\mathrm{DL}} \partial \phi / \partial y$, which is the potential at the Debye length. The boundary conditions for the velocity on the electrode were normal velocity zero and horizontal velocity (in this case $u$ ) given by the electro-osmotic velocity $u_{\mathrm{ACE}}$ calculated from the solution of the electrical potential. The boundary conditions on the symmetry plane were electrical potential zero, normal velocity $u$ zero, and normal derivative of tangential velocity $\nu$ zero. For the remaining boundaries, the normal derivative of the electrical potential was zero and the fluid velocity holds the no-slip condition, $u$ $=\nu=0$.

only to be solved once as a function of frequency for all conductivities by defining the nondimensional frequency $\Omega$ $=\omega \varepsilon L / \sigma \lambda_{D}$, with $L=10^{-4} \mathrm{~m}$ as the typical length of the system. In the second case, the constant phase element impedance of the double layer derived from the experimental results is used, as described in Secs. II and III; $Z_{\mathrm{DL}}$ $=A(i \omega)^{-\beta}$. In this case, the problem must be solved separately for each conductivity. In addition, there is a boundary condition of odd symmetry $\phi=0$ on the plane $x=0$, and a Neumann boundary condition $\partial \phi / \partial n=0$ everywhere else.

Owing to the presence of complex parameters, the problem was solved numerically as a pair of coupled equations for the real and the imaginary parts of the potential. In order to obtain an accurate picture of the frequency-dependent behavior the problem was solved for ten frequencies per decade, evenly spaced logarithmically.

As an illustration, Figs. 7(a) and 7(b) represent the contour lines for the real and imaginary parts of the potential, corresponding to a nondimensional frequency of 8 and applied voltage amplitude $0.5 \mathrm{~V}$.

The electro-osmotic velocity on the surface of the electrodes can then be calculated using Eq. (17). In Fig. 8 the electro-osmotic velocity, calculated numerically for the case $C_{\mathrm{DL}}=\varepsilon / \lambda_{D}$, is plotted as a function of the distance from the electrode edge and nondimensional frequency. Here we have assumed that $\Lambda=1$. It can be seen that the velocity as a function of frequency has a maximum that depends on the distance from the electrode edge: the higher the distance, the lower the frequency. Also, it can be seen that the velocity is negative for values of $x$ close to $x=100 \mu \mathrm{m}$, corresponding to the outer edge of the electrodes, where the liquid is flowing inwards. This is in perfect agreement with experiment 

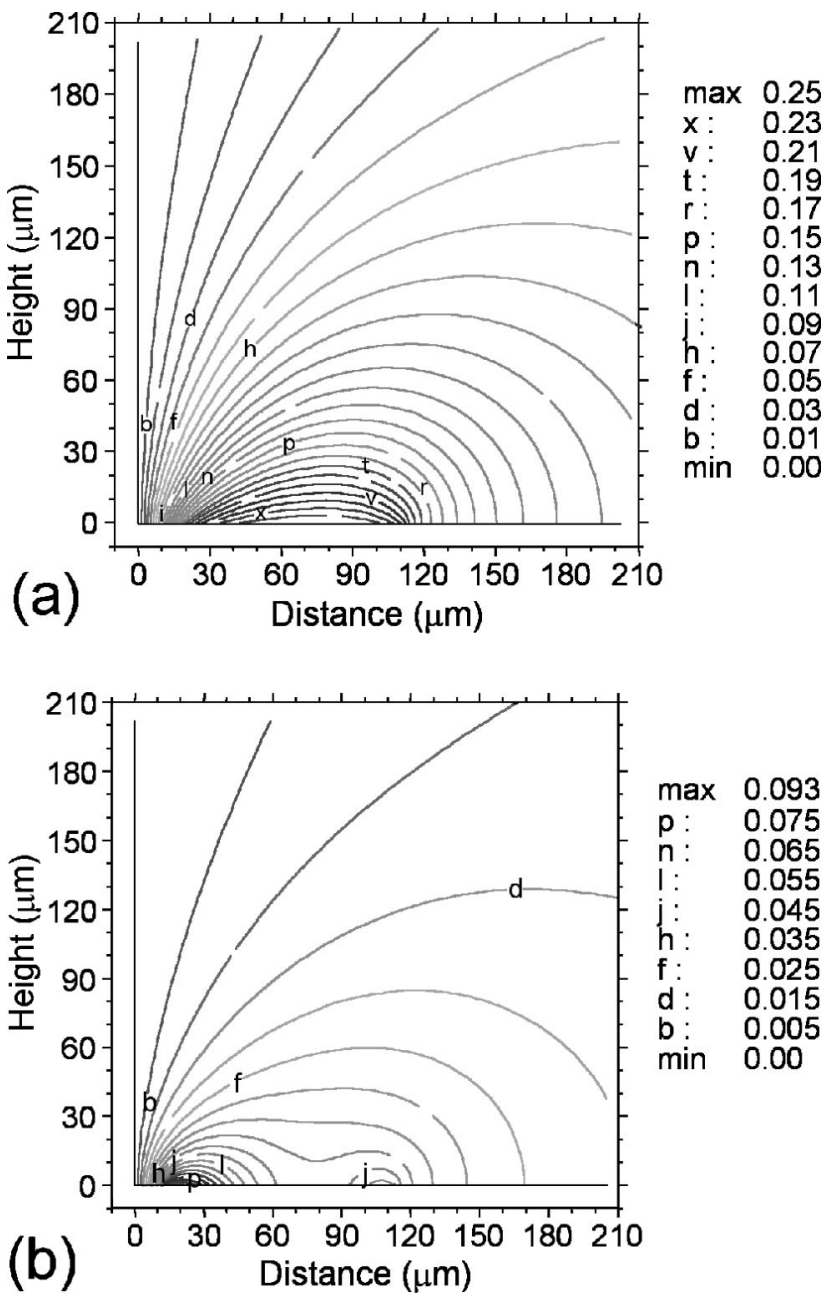

FIG. 7. The electrical potential calculated for a double layer impedance $Z_{\mathrm{DL}}=1 /\left(i \omega C_{\mathrm{DL}}\right)$ shown as separate real (a) and imaginary (b) parts. The voltage applied to the electrode was $0.25 \mathrm{~V}$, corresponding to a potential difference between the electrodes of $0.5 \mathrm{~V}$, and the nondimensional frequency was 8 .

since fluid flow in the opposite direction is observed at the edge of the electrodes farthest from the gap.

Figures 9(a) and 9(b) are comparisons of the experimental velocities and the calculated velocities for electrolytes $A$ and $B$ as a function of frequency, for different positions across the electrode. The curves correspond to an applied voltage amplitude of $0.5 \mathrm{~V}$. For this comparison, the parameter $\Lambda$ was chosen to be 0.25 for case $A$ and 0.24 for case $B$. This factor is the same for all positions.

For the case of the CPA impedance, the system was solved separately for the different conductivities. Figures 10 (a) and 10(b) show the corresponding comparison of the experimental and calculated velocities for the same cases $A$ and $B$ as above, with the same voltage amplitude $0.5 \mathrm{~V}$. Again, to make the comparison, the numerically calculated curves were multiplied by a factor $\Lambda$ smaller than unity $(0.28$ and 0.25 , respectively).

The comparisons demonstrate that better agreement for the frequency of maximum velocity is found when the CPA impedance is used, for both values of conductivity.

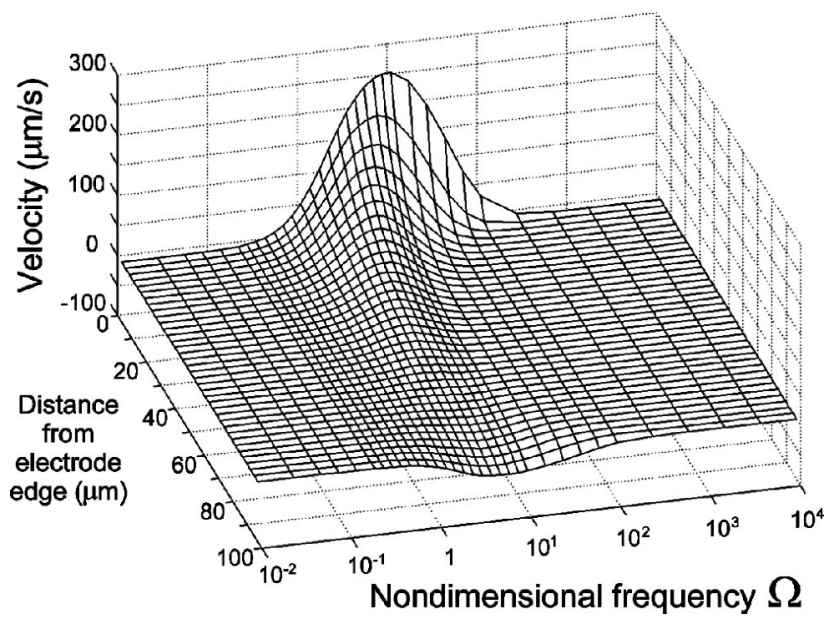

FIG. 8. The horizontal ac electro-osmotic fluid flow at the surface of the electrodes calculated from the electrical potential, plotted as a function of distance from the edge of the electrode and nondimensional frequency. The electrode in this case was $100 \mu \mathrm{m}$ wide, corresponding to the electrodes used for the measurement of the fluid velocity [3]. The amplitude of applied voltage was $0.5 \mathrm{~V}$.

The discrepancy between the Debye-Hückel prediction and the experimental results may be explained because the capacitance of a real double layer is not only due to the diffuse double layer, but also has a component from the compact layer. The fact that matching the experimental and computed velocities requires that the parameter $\Lambda$ be less than 1 is an indication that the voltage drop across the diffuse layer is less than across the whole double layer. A factor $\Lambda$ $\sim 0.25$ suggests that the diffuse layer impedance is a quarter of the total double layer impedance, and the majority of the potential is dropped across the compact layer. This assertion seems to be supported by the experimental impedance data. The measured double layer impedance was assumed to be the sum of the impedance of the diffuse layer and that of a fixed layer, i.e., $Z_{\mathrm{DL}}=Z_{d}+Z_{s}$. As the conductivity of the solution increases, the impedance of the diffuse layer $Z_{d}$ reduces and, for a certain conductivity, should become negligible compared with $Z_{s}$. The ratio between the absolute impedance value for the lowest conductivity solution and the highest conductivity solution is $1.25 \pm 0.10$ in the frequency range of interest. If the double layer impedance for the highest conductivity solution is solely due to the compact layer $\left(Z_{\mathrm{DL}} \approx Z_{s}\right)$, a ratio of 1.25 implies that the potential drop in the diffuse double layer $\Delta \phi_{d}$ for the lowest conductivity solution is around $20 \%$ of the total potential drop, $\Delta \phi_{\mathrm{DL}}$, i.e.,

$$
\left|\frac{\Delta \phi_{d}}{\Delta \phi_{\mathrm{DL}}}\right|=\left|\frac{Z_{d}}{Z_{d}+Z_{s}}\right| \sim 0.2
$$

In other words, the parameter $\Lambda$ in the electro-osmotic velocity equation should be around 0.2 for the lowest conductivity, which is close to the value 0.25 determined by comparison of the experimental and numerical velocity data shown in Fig. 10. The oxide layer increases the distance between the diffuse double layer and the electrode, reducing 

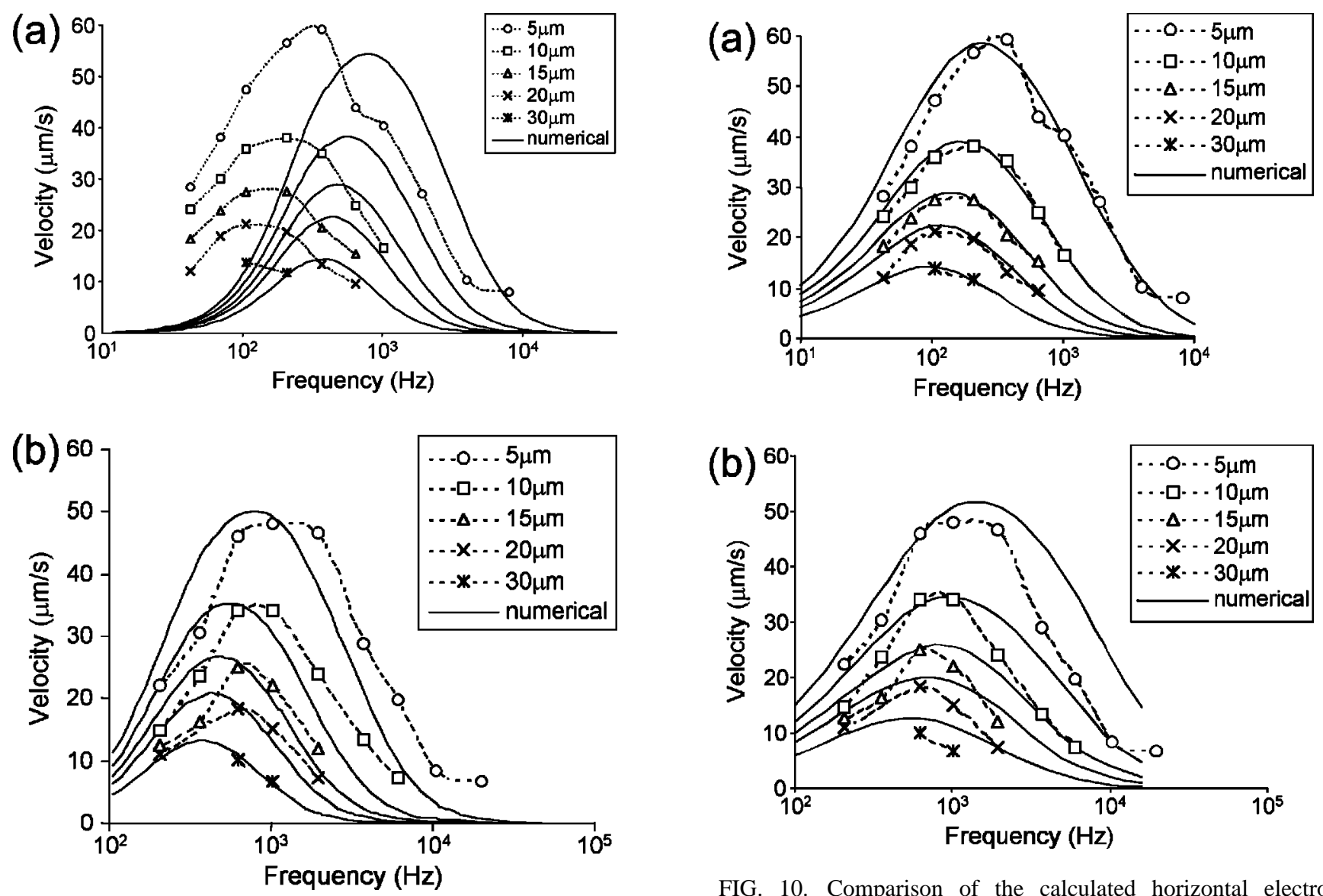

FIG. 9. Comparison of the calculated horizontal electro-osmotic velocity at the surface of the electrodes and the experimental measurements of the fluid velocity across the electrode surface. The data are plotted as a function of frequency (in $\mathrm{Hz}$ ) for several positions on the electrodes and for electrolyte $A$ (a) and electrolyte $B$ (b). The impedance of the double layer was given by $Z_{\mathrm{DL}}$ $=\lambda_{D} /(i \omega \varepsilon)$ and the amplitude of applied voltage was $0.5 \mathrm{~V}$.

the capacitance and increasing the impedance of the double layer. This, in turn, reduces the achievable slip velocity [Eq. (17)].

The fact that for each conductivity and applied voltage, the same value of $\Lambda$ is found for all positions across the electrodes (showing a good agreement in every case for the CPA impedance) suggests that the numerical model correctly predicts the geometry dependence for the liquid velocity. However, the frequency variation of the velocities does not agree completely with the experimental data and could be improved by numerical analysis of a more experimentally realistic three-dimensional system. In addition, in order to predict the conductivity and frequency dependence of ac electro-osmosis from first principles, more theoretical and experimental work is required to understand the physical chemistry of the system.

\section{B. Numerical simulation of bulk fluid flow}

The time-averaged velocity was calculated from Stokes's equations [Eq. (15)] with the following boundary conditions. On the electrodes, the tangential velocity is given by the

FIG. 10. Comparison of the calculated horizontal electroosmotic velocity at the surface of the electrodes and the experimental measurements of the fluid velocity across the electrode surface. The data are plotted as a function of frequency (in $\mathrm{Hz}$ ) for several positions on the electrodes and for electrolyte $A$ (a) and electrolyte $B$ (b). The impedance of the double layer was given by the CPA impedance obtained from the experimental measurements and the amplitude of applied voltage was $0.5 \mathrm{~V}$.

electro-osmotic velocity $u=U$ [from Eq. (17)], with $\Lambda=1$; at the symmetry plane, the tangential velocity satisfies the Neumann boundary condition $\partial v / \partial x=0$; for every other boundary the tangential velocity is zero. The normal velocity is zero over the whole boundary.

Figures 11(a), 11(b), and 11(c) show the predicted streamlines, together with the fluid velocity at each point using the CPA impedance, for conductivity $A$, at frequencies of 100 , 300, and $1000 \mathrm{~Hz}$ and applied voltage $2 \mathrm{~V}$. According to this calculation, the drag of the liquid by the surface stresses produces a roll on top of each electrode. As the frequency increases, the position of the center of the rolls approaches the electrode edges, while the roll size decreases. The liquid moves faster near the inner edges of the electrodes, where the electric field is stronger and changes more sharply.

This velocity distribution can be compared with the experimental streamlines. Figures 12(a), 12(b), and 12(c) show the predicted streamlines superimposed on the composite images of the particle paths, for the same cases as in the previous figures. It can be seen that the numerical computation correctly describes the size and shape of the rolls and the position of the center, the distance of which to the edge de- 

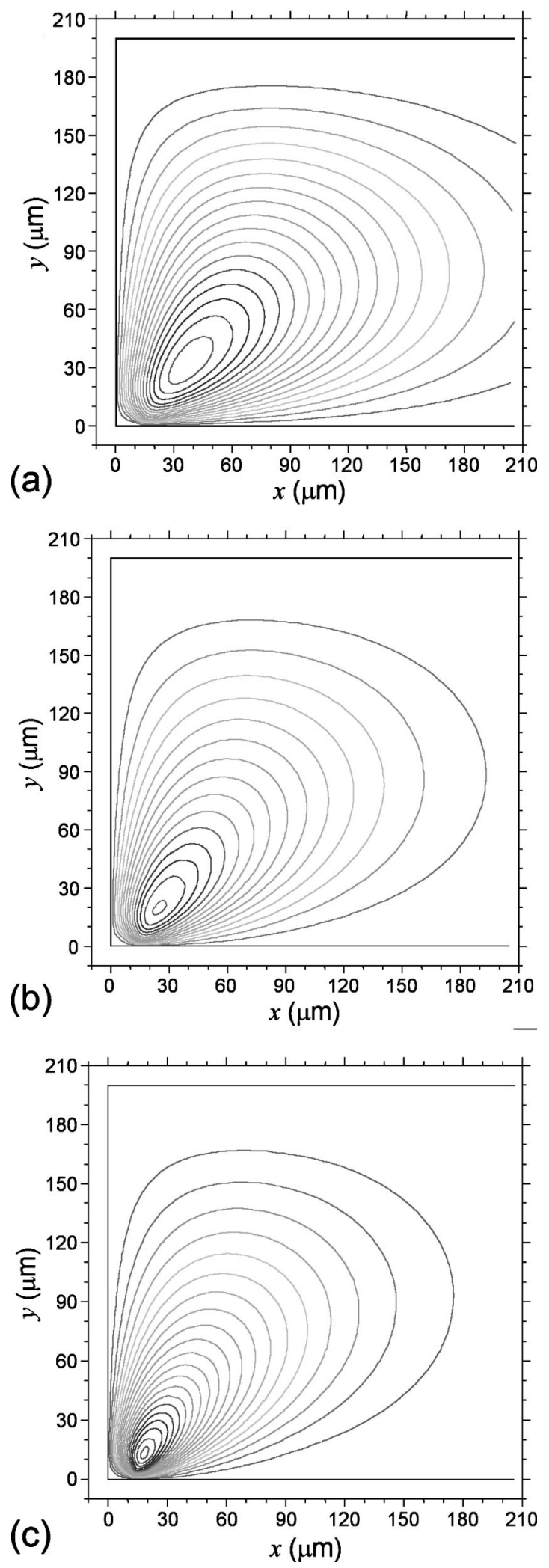

FIG. 11. Numerically computed streamlines for the fluid flow arising from ac electro-osmosis on the surface of $500 \mu \mathrm{m}$ wide electrodes, corresponding to the array used for the observation of the streamlines. The calculations were performed using the CPA impedance, electrolyte $A$ and for the three frequencies used in the experimental observations: (a) 100, (b) 300, and (c) $1000 \mathrm{~Hz}$.
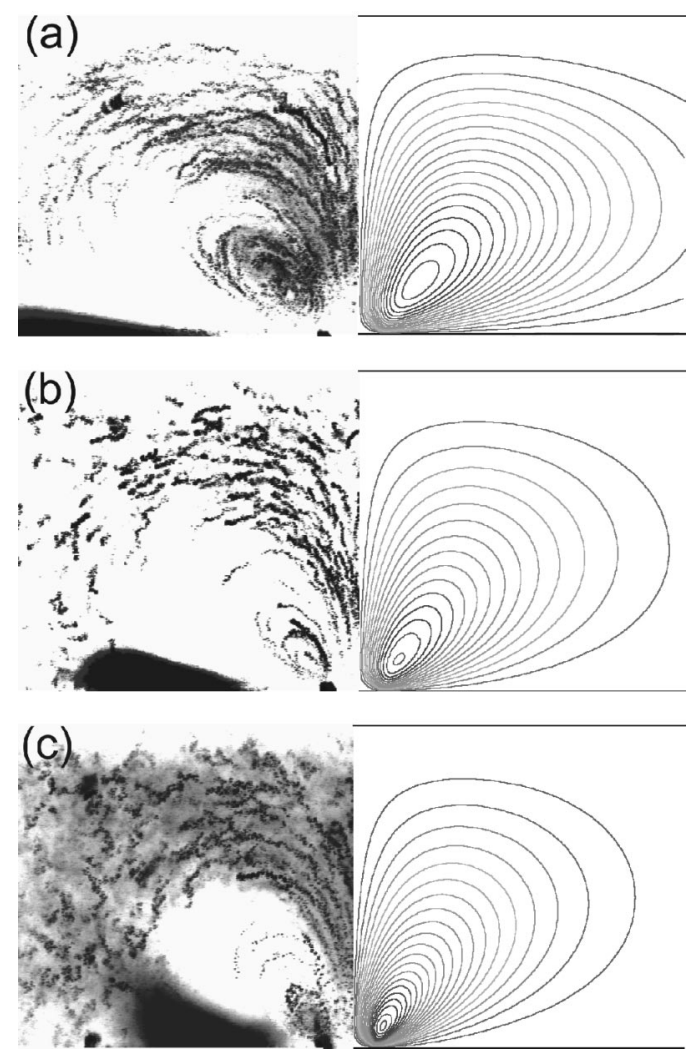

FIG. 12. Comparison of the numerically computed streamlines for $500 \mu \mathrm{m}$ wide electrodes and the experimental streamlines for the three frequencies used in the experimental observations: (a) 100, (b) 300, and (c) $1000 \mathrm{~Hz}$. The experimental images have been inverted.

creases as the frequency increases. This agreement is evidence of the ac electro-osmotic origin of the experimental fluid motion.

A similar calculation for the case of a Debye-Hückel impedance predicts similar rolls, but with a less accurate dependence on frequency.

\section{CONCLUSIONS}

Experimental observations of the streamlines of steady electro-osmotic fluid flow driven by ac fields have been presented. The streamlines were obtained by superimposing images of particle movement in a plane normal to the electrode surface. These experimental streamlines show that the fluid flow is driven at the surface of the electrodes. Experimental measurements of the impedance of the electrical double layer on the electrodes were also presented. The resulting data was then used to model the double layer behavior as a constantphase-angle impedance.

The electrical potential generated by the experimental electrodes as a function of frequency has been numerically calculated using both the Debye-Hückel specific capacitance of the double layer and the experimental CPA impedance. The ac electro-osmotic velocity at the surface of the electrodes has been calculated using the numerical solution of the potential for both cases. A better description of the velocity dependence on frequency was obtained by using the 
CPA impedance. The bulk fluid flow driven by this surface velocity was also calculated numerically as a function of frequency and good agreement was found between the numerical and experimental streamlines, demonstrating that the fluid motion is caused by ac electroosmosis.

To match the numerical and experimental velocity magnitudes, the value of the parameter $\Lambda$ was $\approx 0.25$. This is because of the existence of a layer of oxide layer between the titanium electrode and the electrolyte, reducing the potential drop across the diffuse layer and, in turn, reducing the expected velocity magnitude.

This fluid flow described in this paper generally occurs in a system involving nonuniform fields generated by micro- electrodes at frequencies of the order of kilohertz. The results presented in this paper could be relevant to the problem of particle-particle interaction seen on top of electrodes in ac fields such as described in Ref. [30].

\section{ACKNOWLEDGMENTS}

The authors would like to acknowledge the European Union for financial support to N.G.G. [Contract No. BIO4CT98-5010 (DG12-SSM)] and the Spanish government agency Dirección General de Ciencia y Tecnologia for financial support under Contract No. BFM2000-1056.
[1] A. Ramos, H. Morgan, N. G. Green, and A. Castellanos, J. Colloid Interface Sci. 217, 420 (1999).

[2] A. Ramos, H. Morgan, N. G. Green, and A. Castellanos, J. Electrost. 47, 71 (1999).

[3] N. G. Green, A. Ramos, A. González, H. Morgan, and A. Castellanos, Phys. Rev. E 61, 4011 (2000).

[4] A. González, A. Ramos, N. G. Green, H. Morgan, and A. Castellanos, Phys. Rev. E 61, 4019 (2000).

[5] A. Ramos, H. Morgan, N. G. Green, and A. Castellanos, J. Phys. D 31, 2338 (1998).

[6] N. G. Green, A. Ramos, A. González, A. Castellanos, and H. Morgan, J. Electrost. 53, 71 (2001).

[7] G. Fuhr, R. Hagedorn, T. Müller, W. Benecke, and B. Wagner, J. Microelectromech. Syst. 1, 141 (1992).

[8] A. B. D. Brown, C. G. Smith, and A. R. Rennie, Phys. Rev. E 63, 016305 (2001).

[9] A. Ajdari, Phys. Rev. E 61, R45 (2000).

[10] M. Trau, D. A. Saville, and I. A. Aksay, Langmuir 13, 6375 (1997).

[11] S-R. Yeh, M. Seul, and B. I. Shraiman, Nature (London) 386, 57 (1997).

[12] P. J. Sides, Langmuir 17, 5791 (2001).

[13] R. J. Hunter, Zeta Potential in Colloid Science (Academic, New York, 1981).

[14] N. G. Green, A. Ramos, A. González, A. Castellanos, and H. Morgan, J. Phys. D 33, L13 (2000).
[15] M. Aziz-Kerrzo, K. G. Conroy, A. M. Fenelon, S. T. Farrell, and C. B. Breslin, Biomaterials 22, 1531 (2001).

[16] N. G. Green, A. Ramos, and H. Morgan, J. Phys. D 33, 632 (2000).

[17] T. B. Jones, Electromechanics of Particles (Cambridge University Press, New York, 1995).

[18] Y. Solomentsev, M. Bohmer, and J. L. Anderson, Langmuir 13, 6058 (1997).

[19] S. H. Liu, Phys. Rev. Lett. 55, 529 (1985).

[20] J. B. Bates, T. T. Chu, and W. T. Stribling, Phys. Rev. Lett. 60, 627 (1988).

[21] T. C. Halsey and M. Leibig, Phys. Rev. A 43, 7087 (1991).

[22] Z. Kerner and T. Pajkossy, J. Electroanal. Chem. 448, 139 (1998).

[23] Z. Kerner and T. Pajkossy, Electrochim. Acta 46, 207 (2000).

[24] H. H. Sun and B. Onaral, IEEE Trans. Biomed. Eng. BME-30, 399 (1983).

[25] H. P. Schwan, Ann. Biomed. Eng. 20, 269 (1992).

[26] J. Gunning, D. Y. C. Chan, and L. R. White, J. Colloid Interface Sci. 170, 522 (1995).

[27] V. G. Levich, Physicochemical Hydrodynamics (Prentice-Hall, Englewood Cliffs, NJ, 1962).

[28] http://www.pdesolutions.com

[29] G. Backstrom, Fluid Dynamics by Finite Element Analysis (Studentlitteratur, Lund, 1999).

[30] J. Kim, S. A. Guelcher, S. Garoff, and J. L. Anderson, Adv. Colloid Interface Sci. 96, 131 (2002). 